\title{
Clinicopathologic and Dermoscopic Features of Acquired Perforating Dermatosis: A Case Report
}

\author{
Jay-V James G. Barit, ${ }^{1}$ Felix Paolo J. Lizarondo ${ }^{1}$ and Eileen Liesl A. Cubillan² \\ ${ }^{1}$ Section of Dermatology, Department of Medicine, Philippine General Hospital, University of the Philippines Manila \\ ${ }^{2}$ Section of Dermatology, Department of Medicine, College of Medicine and Philippine General Hospital, University of the Philippines Manila
}

\begin{abstract}
Acquired perforating dermatosis represents one of the perforating skin disorders showing transepidermal elimination of dermal contents. Dermoscopy showing concentric zones of a central keratotic plug, white-gray structureless area and peripheral brown pigmentation may aid in diagnosis and in sample selection for biopsy.
\end{abstract}

Key Words: Acquired Perforating Dermatosis, Type 2 Diabetes Mellitus, kidney failure, chronic, dermoscopy

\section{INTRODUCTION}

Poster presented at the 2018 Research Poster Presentation of the Expanded Hospital Research Office (EHRO), October 8-12, 2018, Philippine General Hospital, Manila, Philippines.

E-poster presented at the $41^{\text {st }}$ Annual Convention of the Philippine Dermatological Society, November 7-9, 2018, EDSA Shangri-La, Mandaluyong City, Philippines.

Corresponding author: Jay-V James G. Barit, MD

Section of Dermatology

Department of Medicine

Philippine General Hospital

University of the Philippines Manila

Taft Avenue, Manila 1000, Philippines

Telephone: +632 5548400 extension 5105/5106

Email: jayvjamesbarit@gmail.com
Perforating dermatoses are skin disorders that represent transepidermal elimination of dermal connective tissue. Classically they have been described as four distinct "primary" disorders depending on clinicohistopathologic features and type of material eliminated: 1) reactive perforating collagenosis (RPC) - rare childhood disorder with collagen elimination, 2) elastosis perforans serpiginosa (EPS) elastin elimination beginning in childhood, 3) perforating folliculitis (PF) - elimination through a hair follicle, and 4) Kyrle disease - elimination of keratotic material). ${ }^{1} \mathrm{~A}$ similar papular eruption with transepidermal elimination of any of the aforementioned dermal contents has been observed in patients with diabetes mellitus or renal disease and is termed acquired perforating dermatosis (APD). Currently, APD encompasses Kyrle disease and all other acquired forms of RPC, EPS and PF, mostly among patients with underlying diabetes mellitus or chronic kidney disease. APD usually presents with dome-shaped papules or nodules with a central crusted crater. ${ }^{1,2}$ Often the lesions are pruritic, with possible koebnerization after scratching, and commonly involve the trunk and extremities. Its cause is poorly understood but trauma from scratching and a microvasculopathy are suggested triggers for the degeneration of dermal contents and their transepidermal elimination. ${ }^{3}$ This case report describes the clinical, histopathologic and dermoscopic features of a case of APD in a Filipino patient with both diabetes mellitus and chronic kidney disease. Distinct dermoscopic findings may aide site selection for biopsy to properly establish its diagnosis.

\section{CASE REPORT}

A 47-year-old Filipino male presented with a four-year history of initially few sporadic erythematous papules that heal with hyperpigmentation. The lesions were occasionally 

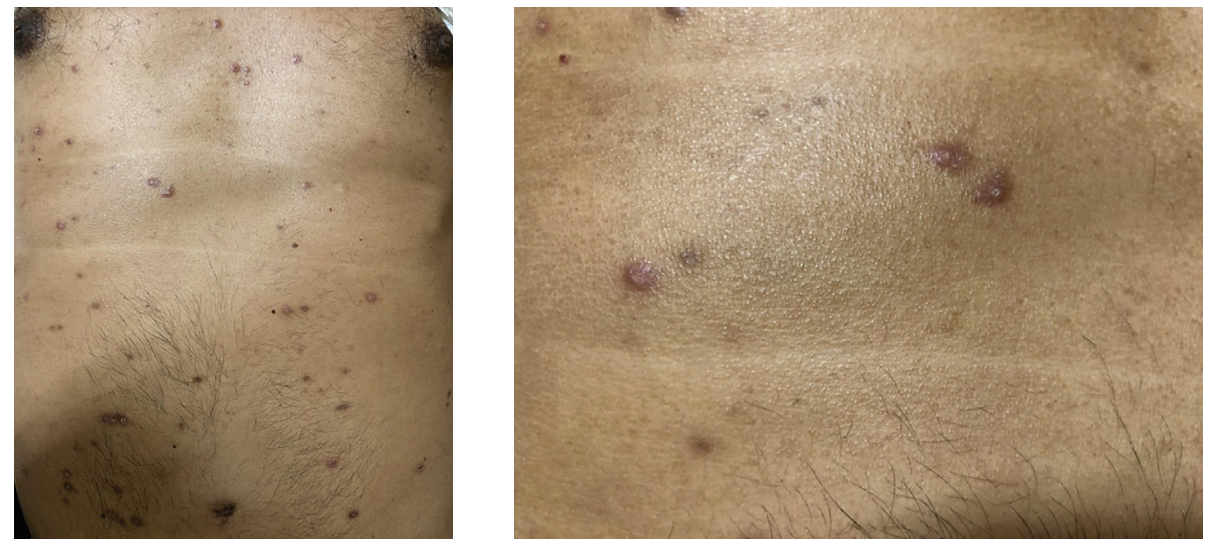

Figure 1. Multiple round, brown, firm scaly papules some with a central keratotic plug in the abdomen.
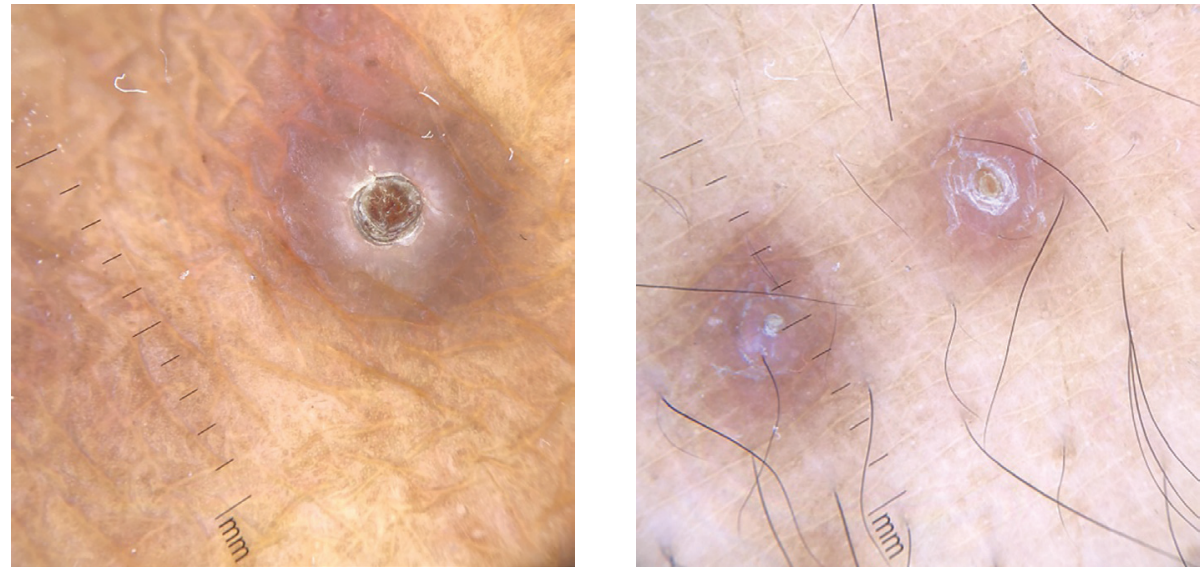

Figure 2. Dermoscopy showing three concentric zones: central keratotic plug, a whitish structureless area and a peripheral zone of hyperpigmentation (10x).

tender, and some were observed to spontaneously allegedly ulcerate and heal. There was associated pruritus. No topical or oral medications were used. He was a known Type 2 diabetic for nine years and hypertensive for three years, and was poorly compliant to oral maintenance medications. He was diagnosed one year ago with chronic kidney disease and had been on hemodialysis twice a week. On physical examination, there were multiple round, brown, firm scaly papules, some with a central keratotic plug in the abdomen and extremities (Figure 1). Dermoscopy of the lesions using a manual dermoscope (DermLite DL4; 3Gen, San Capistrano, CA, USA) through a 12-megapixel mobile phone camera showed three zones: a central white or brown keratotic plug; a surrounding whitish-gray structureless area; and a peripheral brown pigmentation (Figure 2). A 4-mm punch biopsy of an abdominal papule was taken. Routine histology showed epidermal hyperplasia, as well as transepidermal elimination of degenerated collagen fibers with a central basophilic plug consisting of keratin, collagen and a mixed inflammatory infiltrate (Figure 3). The patient was given tretinoin 0.05\% cream applied once daily for his lesions.

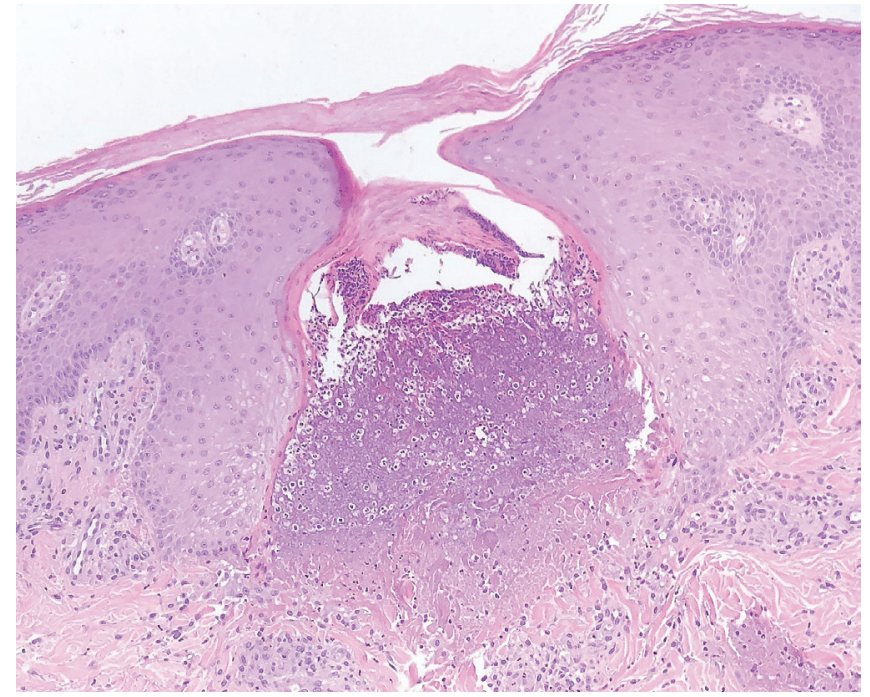

Figure 3. High power magnification showing epidermal hyperplasia, transepidermal elimination of degenerated collagen fibers with a central basophilic plug consisting of keratin, collagen and a mixed inflammatory infiltrate (40x). 


\section{DISCUSSION}

The exact prevalence and incidence of APD is not known: a recent retrospective review of 31 patients in Spain between 2002 to 2014 showed a low incidence of 2.53 cases per 100,000 annually, but the authors suggest that the disease maybe more common but just potentially misdiagnosed. ${ }^{4}$ Various retrospective reviews in different centers examining the associated systemic disorders with APD showed diabetes mellitus and chronic kidney disease as the most associated conditions. ${ }^{4,5,6}$ The histopathologic pattern for RPC (i.e., cup-shaped invagination of the epidermis with a plug of necrotic inflammatory debris and collagen bundles) is the most common histologic pattern in APD patients. ${ }^{4,5}$ An earlier study though showed the classic pattern of Kyrle's disease (showing keratotic debris that is negative for collagen and elastic fibers) to be the most common finding. ${ }^{6}$ In all of these studies, all histolopathologic patterns seen in primary perforating dermatoses had been identified in APD lesions. Thickened blood vessels seen with periodic acid-Schiff staining in some cases suggested a vasculopathic effect among diabetic patients with APD. ${ }^{3}$

The current theories on its pathogenesis lie on microtrauma caused by scratching which may be responsible for focal degeneration of dermal fibers. Glycation of proteins, in the setting of diabetes mellitus for example, alter collagen fibers, and with microtrauma may trigger transepidermal elimination of these modified dermal proteins. ${ }^{7}$ In a study examining cell cultures, it has been demonstrated that involucrin and keratin 10 were enhanced in keratinocytes exposed to advanced glycation end product-modified collagens I and III, inducing terminal differentiation of keratinocytes. The authors hypothesized that through scratching, this process allowed for the upward migration of keratinocytes, together with the modified collagen, leading to transepidermal elimination. ${ }^{8} \mathrm{~A}$ more recent study examining receptor for advanced glycation end products (RAGE) in acquired RPC showed that RAGE expression was more intense in RPC lesions compared to controls in the endothelium, inflammatory cells and fibroblasts in immunostained biopsies, independent of diabetes. ${ }^{9}$ Overexpression of transforming growth factor-3 and extracellular matrix proteins had been detected in the epidermal depressions of APD lesions; this signaling pathway for wound repair maybe an antecedent event in the resolution of APD lesions. ${ }^{10}$

Dermoscopy of APD lesions show a 3-zonal concentric pattern. The center is characterized by bright whitishbrownish scales, which correspond histologically to keratotic plugs. A structureless whitish-grey area surrounds the central crusts and on biopsy appears as acanthosis, hypergranulosis and post-inflammatory pigmentation. A peripheral, brown, delicate reticulate pigmentation surrounds the structureless area, reflecting basal layer pigmentation on histology. ${ }^{11}$ These findings may help differentiate APD lesions from its more common differential prurigo nodularis, which on dermoscopy appears as a "white starburst pattern" (peripheral radially arranged whitish lines or whitish halo) on a brown or red background, as well as hyperkeratosis or scaling, crusts and erosions. ${ }^{12}$ Dermoscopy may then aid lesion selection for biopsy, in order to properly establish the diagnosis of APD and select proper management.

Treatment of APD can be challenging but symptomatic management of pruritus is needed. Topical antipruritics (urea-, capsaicin-based formulations), antihistamines and phototherapy are used in pruritus. Keratolytics, such as salicylic acid, urea and tretinoin could be applied under occlusion. Systemic and intralesional corticosteroids, as well as systemic retinoids may also be used. Reports on allopurinol are conflicting; and isolated reports using hydroxychloroquine, doxycycline, dapsone and methotrexate have been documented. ${ }^{7}$

\section{CONCLUSION}

Acquired perforating dermatosis is a distinct dermatologic disorder common in patients with diabetes mellitus and chronic kidney disease presenting as hyperkeratotic papules with associated pruritus. As a highly variable histopathologic entity, its proper recognition clinically allows for management. The most common histopathologic pattern of APD is transepidermal elimination of collagen fibers seen in reactive perforating collagenosis. Distinct dermoscopic findings of a three-zone central hyperkeratosis, structureless area and reticulate hyperpigmentation may aid in clinical diagnosis as well as biopsy site selection.

\section{Disclaimer}

The views expressed in this article are the authors' own and do not reflect the views of the institution.

\section{Statement of Authorship}

All authors approved the final version submitted.

\section{Author Disclosure}

All authors declared no conflicts of interest.

\section{Funding Source}

This paper was funded by the authors. No external funding agency.

\section{REFERENCES}

1. Farrell AM. Acquired perforating dermatosis in renal and diabetic patients. Lancet. 1997 ;349(9056):895-6.

2. Rapini RP, Herbert AA, Drucker CR. Acquired Perforating Dermatosis: Evidence for combined transepidermal elimination of both collagen and elastic fibers. Arch Dermatol. 1989;125(8):1074-8

3. Hong S-B, Park J-H, Ihm C-G, Kim N-I. Acquired Perforating Dermatosis in Patients with Chronic Renal Failure and Diabetes Mellitus. J Korean Med Sci. 2004;19(2):283-8. 
4. García-Malinis A, Sánchez EDV, Sánchez-Salas M, Prado ED, Coscojuela C, Gilaberte Y. Acquired perforating dermatosis: clinicopathological study of 31 cases, emphasizing pathogenesis and treatment. J Eur Acad Dermatol Venereol. 2017;31(10):1757-63. doi: 10.1111/jdv.14220.

5. Kim SW, Kim MS, Lee JH, Son S-J, Park KY, Li K, et al. A Clinicopathologic Study of Thirty Cases of Acquired Perforating Dermatosis in Korea. Ann Dermatol. 2014 ;26(2):162-71. doi: 10.5021/ad.2014.26.2.162.

6. Saray Y, Seckin D, Bilezikci B. Acquired perforating dermatosis: clinicopathological features in twenty-two cases. J Eur Acad Dermatol Venereol. 2006;20(6):679-88.

7. Wagner G, Sachse MM. Acquired reactive perforating dermatosis. J Dtsch Dermatol Ges. 2013 Aug;11(8):723-9, 723-30. doi: 10.1111/ ddg.12131.

8. Fujimoto E, Kobayashi T, Fujimoto N, Akiyama M, Tajima S, Nagai R. AGE-Modified Collagens I and III Induce Keratinocyte Terminal Differentiation through AGE Receptor CD36: EpidermalDermal Interaction in Acquired Perforating Dermatosis. J Invest Dermatol. 2010 Feb;130(2):405-14. doi: 10.1038/jid.2009.269.
9. Akoglu G, Sungu N, Karaismailoglu E, Aktas A. Expression of the receptor for advanced glycation end products in acquired reactive perforating collagenosis. Indian J Dermatol Venereol Leprol. 2017;83(4):432-5. doi: 10.4103/ijdvl.IJDVL_113_16.

10. Gambichler T, Birkner L, Stücker M, Othlinghaus N, Altmeyer P, Kreuter A. Up-regulation of transforming growth factor- $\beta 3$ and extracellular matrix proteins in acquired reactive perforating collagenosis. J Am Acad Dermatol. 2009 Mar;60(3):463-9. doi: 10.1016/j.jaad.2008.06.006.

11. Ramirez-Fort MK, Khan F, Rosendahl CO. Acquired perforating dermatosis: a clinical and dermatoscopic correlation. Dermatology Online Journal [Internet].2013 [cited 2013 Jan1];19(7). Available from: https://escholarship.org/uc/item/7q40n20h

12. Errichetti E, Piccirillo A, Stinco G. Dermoscopy of prurigo nodularis. J Dermatol. 2015 ;42(6):632-4. doi: 10.1111/1346-8138.12844.

\section{The Acta Medica Philippina is now accepting limited advertising for its front and back cover (colored), as well as for available spaces in some of its pages, as appropriate. For inquiries and submission of proposals, please e-mail us at actamedicaphilippina@yahoo.com}

